SANDIA REPORT

SAND95-1352 • UC-706

Unlimited Release

Printed September 1997

\section{RECEIVED}

SEP 151997

OSTI

\title{
Issues in Computational Fluid Dynamics Code Verification and Validation
}

William L. Oberkampf, Frederick G. Blottner

\section{Prepared by}

Sandia National Laboratories

Albuquerque, New Mexico 87185 and Livermore, California 94550

Sandia is a multiprogram laboratory operated by Sandia Corporation, a Lockheed Martin Company, for the United States Department of Energy under Contract DE-AC04-94AL85000.

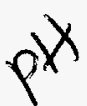

DISTRBDTON OF THS DOCUMENT IS UNIMIE.

Approved for public release; distribution is unlimited.

\section{in Sandia National Laboratories}


Issued by Sandia National Laboratories, operated for the United States Department of Energy by Sandia Corporation.

NOTICE: This report was prepared as an account of work sponsored by an agency of the United States Government. Neither the United States Government nor any agency thereof, nor any of their employees, nor any of their contractors, subcontractors, or their employees, makes any warranty, express or implied, or assumes any legal liability or responsibility for the accuracy, completeness, or usefulness of any information, apparatus, product, or process disclosed, or represents that its use would not infringe privately owned rights. Reference herein to any specific commercial product, process, or service by trade name, trademark, manufacturer, or otherwise, does not necessarily constitute or imply its endorsement, recommendation, or favoring by the United States Government, any agency thereof, or any of their contractors or subcontractors. The views and opinions expressed herein do not necessarily state or reflect those of the United States Government, any agency thereof, or any of their contractors.

Printed in the United States of America. This report has been reproduced directly from the best available copy.

Available to DOE and DOE contractors from

Office of Scientific and Technical Information

P.O. Box 62

Oak Ridge, TN 37831

Prices available from (615) 576-8401, FTS 626-8401

Available to the public from

National Technical Information Service

U.S. Department of Commerce

5285 Port Royal Rd

Springfield, VA 22161

NTIS price codes

Printed copy: A03

Microfiche copy: A01 


\title{
Issues in Computational Fluid Dynamics
}

\section{Code Verification and Validation}

\author{
William L. Oberkampf and Frederick G. Blottner \\ Aerosciences and Compressible Fluid Dynamics Dept. \\ Mail Stop 0825 \\ Sandia National Laboratories \\ P. O. Box 5800 \\ Albuquerque, New Mexico 87185-0825
}

\begin{abstract}
A broad range of mathematical modeling errors of fluid flow physics and numerical approximation errors are addressed in computational fluid dynamics (CFD). It is strongly believed that if CFD is to have a major impact on the design of engineering hardware and flight systems, the level of confidence in complex simulations must substantially improve. To better understand the present limitations of CFD simulations, a wide variety of physical modeling, discretization, and solution errors are identified and discussed. Here, discretization and solution errors refer to all errors caused by conversion of the original partial differential, or integral, conservation equations representing the physical process, to algebraic equations and their solution on a computer. The impact of boundary conditions on the solution of the partial differential equations and their discrete representation will also be discussed. Throughout the article, clear distinctions are made between the analytical mathematical models of fluid dynamics and the numerical models. Lax's Equivalence Theorem and its frailties in practical CFD solutions are pointed out. Distinctions are also made between the existence and uniqueness of solutions to the partial differential equations as opposed to the discrete equations. Two techniques are briefly discussed for the detection and quantification of certain types of discretization and grid resolution errors.
\end{abstract}




\section{Acknowledgements}

The authors thank Daniel Aeschliman, Robert Cochran, Steven Kempka, and Mary Walker of Sandia National Laboratories for helpful suggestions and discussions during preparation of this article. We also appreciate the comments and suggestions provided by the reviewers for the AIAA Journal. This work was performed at Sandia National Laboratories, which is operated by Lockheed Martin Corp. for the U. S. Department of Energy under contract No. DE-AC0494AL85000. 


\section{DISCLAMMER}

Portions of this document may be illegible in electronic image products. Images are produced from the best avallable original document. 



\section{Contents}

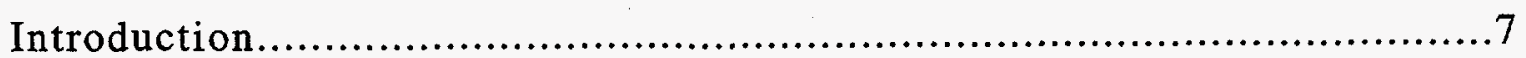

Sources of Simulation Error....................................................... 8

Physical Modeling Error........................................................ 9

Partial Differential Equations of Fluid Dynamics.................................... 10

Auxiliary Physical Models.................................................... 12

Boundary Conditions for the Partial Differential Equations............................. 15

Discretization and Solution Errors................................................. 17

Discretization of the Partial Differential Equations...................................... 18

Discretization of the Auxiliary Physical Models........................................20

Discretization and Implementation of Boundary Conditions...........................20

Solution of the Discrete Equations............................................. 21

Detection and Estimation of Errors...............................................22

Discretization Errors........................................................... 22

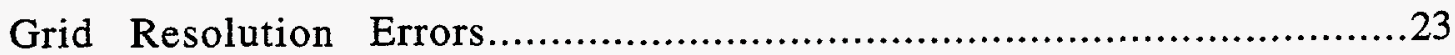

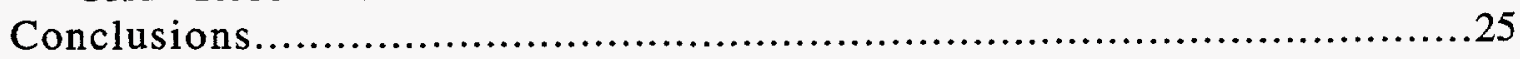

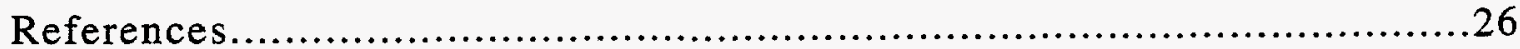


Intentionally Left Blank

$-6-$ 


\section{Introduction}

Quantifying the level of confidence, or accuracy, of computational fluid dynamics (CFD) codes and numerical simulations has recently received increased levels of attention in the research and engineering application literature. Both CFD researchers and users of CFD codes are asking more critical and detailed questions concerning the accuracy and range of applicability of CFD code predictions. This trend is welcomed because it shows that CFD is beginning to mature from a research exercise to a useful tool that impacts the design of engineering hardware and flight systems. Ten years ago the National Research Council committee chaired by Richard Bradley ${ }^{1}$ summarized the two pertinent stages of CFD development. Stage IV, "Learning to Use Effectively," is characterized by "changing the engineering process" and "value exceeds expectation." Stage V, "Mature Capability," is characterized by "entrenched, cost effective" process. Delineation between these two stages is marked by "Most analysis done without supporting experimental comparisons." Using these definitions, most of us would agree that CFD is definitely not in Stage V.

A clear parallel can be drawn between the progress of CFD capability and the history of numerical solutions of ordinary differential equations (ODEs), specifically, the simulation of electrical analog circuits. Until twenty or thirty years ago, the response of complex analogue circuits was determined by bread-board experiments. Since that time, the numerical simulation capability, in both hardware and software, for solving ordinary differential equations has completely changed the technology and design process. Designers now do few, if any, breadboard experiments, but they fundamentally rely on numerical simulation of the response of circuits. ODE solution codes are clearly in Stage V, as defined by the National Research Council. Today the key issues in circuit simulation are related to characterization of electrical components as a function of voltage, current, temperature, electric field, and environmental parameters. Progress to Stage V for ODE solvers has been rapid for three reasons. First, the mathematical complexity of solving ODEs is, in general, miniscule compared to partial differential equations (PDEs). Second, the complexity of fluid flow physics contained in the Navier-Stokes equations is significantly more involved than the physics associated with ODEs. And third, geometry does not enter the problem for one independent variable of ODEs, whereas in PDEs, geometry is fundamental to the description of the problem.

During the last few years, new technology development concepts and terminology have arisen that take advantage of the increased capability of numerical simulations. Terminology such as "virtual prototyping" and "virtual testing" are now being used by those in engineering development to describe numerical simulation for design, evaluation, and "testing" of new hardware and even entire systems. This trend is driven by increasing competition in many markets, such as aircraft, automobiles, propulsion systems, and consumer products. The need to decrease the time and cost of bringing products to market is intense. Another reason for this new trend is the high cost and time required for testing laboratory or field components and complete systems. The safety aspects of the product or system represent an important, sometimes dominant, element of testing or validating numerical simulations. The potential legal and liability costs of hardware failures can be staggering to a company, the environment, or the public. Examples of increasing levels of impact are: wave induced structural failure of an 
offshore oil platform, fire spread in a commercial aircraft accident, pressure vessel failure of a nuclear power plant, and accidental detonation of a nuclear weapon in a fire environment. In CFD research simulations, however, the impact of an inaccurate or misleading numerical simulation in a conference paper or a journal article is commonly nil.

The terminology, philosophy and methodology of building confidence in CFD code predictions is proving to be a very difficult and complex issue. The issues have been discussed and debated in the literature and within various engineering societies for several years. The Institute of Electrical and Electronics Engineers (IEEE) ${ }^{2}$ and the American Nuclear Society 3 first studied the terminology and procedures of code verification and validation. In the late 1980's a NASA ad hoc committee was formed and they produced a detailed definition of code calibration and validation ${ }^{4}$. In the early 1990 's the International Organization for Standardization (ISO) ${ }^{5}$, the American Institute of Aeronautics and Astronautics Committee on Standards, and the American Society of Mechanical Engineers also became involved in the issue. Because of the far reaching effects on hardware design, economic competitiveness, government contracting, commercial software, product liability, etc., we believe these matters should be debated in these forums. For a review of some perspectives on this issue see Refs. 6-8.

The present article avoids the debate on terminology and concentrates on the fundamental issues of mathematical modeling of fluid dynamics and the numerical solution of the resulting equations. This article uses the following definitions: verification is "solving the equations right"; and validation is "solving the right equations." 7,9-11 The essence of these simple definitions is that verification only deals with the accuracy of the solution of the assumed mathematical model. Validation deals with the accuracy of the solution as it relates to the real world, i. e., experimental data. Within these broad terms this article addresses a wide variety of physical modeling, discretization, and solution errors. By physical modeling is meant the analytical mathematical equations that are used to describe a physical process or characteristic. Discretization errors refer to all errors caused by conversion of the original partial differential, or integral, conservation equations representing the physical process, to algebraic equations.

Boundary conditions for both the partial differential equations and the discretized equations will be discussed. Throughout the article, clear distinctions are made between the analytical mathematical models of fluid dynamics and the numerical models. Lax's Equivalence Theorem, which is concerned with the convergence of the solution of the discrete equations to the solution of the partial differential equations, is discussed. Comments are also made concerning the existence and uniqueness of solutions for both the partial differential equations and the discrete equations. A few techniques are suggested for the detection and quantification of certain types of discretization and solution errors. The goal of this article is to raise, or resurrect, many fundamental issues and questions that impact simulation verification and validation; not to suggest solutions for all of them.

\section{Sources of Simulation Error}

To assess conceptually the accuracy of CFD solutions, one must recognize that CFD 
regularly addresses significantly more complex mathematical issues than analytical methods. Whether they are dealt with accurately or not, is the topic of this article. The accuracy required of a simulation, however, depends on the requirements of the particular simulation. Most researchers and users of CFD codes view CFD as an extension, or outgrowth, of traditional analytical methods in mathematical physics. Analytical methods, i.e., closed form, exact or perturbation solutions to the equations of interest, are built on well defined, very reproducible, and rigorous methods of mathematical analysis. As is well known, the dominant shortcoming of analytical methods is that they address a much narrower range of fluid dynamics than computational methods. The accuracy of analytical predictions fundamentally depends on the accuracy of the mathematical model of the physics. The rigor and accuracy of the mathematical methods is rarely an issue. CFD prediction accuracy, on the other hand, additionally depends on the equivalence of the discrete model to the continuum model and the accuracy of the solution of the discrete model. We have become convinced that clearly separating the mathematics of the discrete model and the continuum model fundamentally aids in identifying sources of error.

To better understand the accuracy of CFD predictions, the fundamental sources of errors, or inaccuracies, must be identified and addressed. Error sources in numerically simulating physical phenomena described by partial differential equations can be grouped into four broad categories:

- Physical modeling errors

- Discretization and solution errors

- Programming errors

- Computer round-off errors

The present article delineates and discusses the first two types of errors, but ignores programming and computer round-off errors. The first two categories are subdivided further in the following sections and each is discussed. The above categories are appropriate for any conservation laws described by partial differential equations, $e . g$., heat conduction, solid dynamics, structural dynamics, electrodynamics, and neutron transport. The present discussion, however, will be limited to continuum fluid dynamics with emphasis on Newtonian fluids. Other approaches in fluid dynamics, such as molecular dynamics, direct simulation Monte Carlo. and lattice gas methods, are not considered in this article.

\section{Physical Modeling Errors}

Physical modeling errors are those caused by inaccuracies in the mathematical model of the physics. These errors are completely separate from numerical ones. The errors in question are further subdivided into three additional categories associated with: the partial differential equations describing the flow; the auxiliary (or closure) physical models; and the boundary 
conditions for all of the partial differential equations. All three of these sub-categories are discussed to stress the evaluation of physical modeling limitations, as opposed to numerical solution limitations, when conducting a CFD simulation. Too often, difficulties in detecting and eliminating CFD simulation errors, whether in code V\&V activities or in day-to-day simulations, are due to a failure to consider each error source separately. Indeed, in large computer codes most code developers seldom segregate the continuum physical model from the discrete model. For example, a new boundary condition may be tried in the code without any thought of how this might relate to a change in the continuum boundary conditions.

\section{Partial Differential Equations of Fluid Dynamics}

The governing equations for fluid flow can be formulated with various assumptions and the analyst is usually interested in using the simplest form that is appropriate for the problem being solved. The starting point for the following discussion is the compressible form of the unsteady Euler equations and then the governing equations are expanded to include more complex physics. As the complexity of the physics increases, more information is required in the modeling and more uncertainty is introduced into the partial differential equations. The following list is not comprehensive, but does address the more common areas of fluid dynamics.

Inviscid Flow: The partial differential equations that govern the flow of a compressible, inviscid, continuum fluid can be developed from the conservation laws of mass, momentum, and energy. The resulting equations are expected to be valid for all flows except when the size of the flow region of interest is of the same order as a characteristic length of the molecular mean free path. These same equations can be developed with the Chapman-Enskog expansion method from the kinetic theory of gases. ${ }^{12}$ The first term in the expansion gives the Euler equations while the second term in the expansion gives the Navier-Stokes equations. The Chapman-Enskog approach is valid for flows that are collision-dominated and is not appropriate for rarefied flows as previously indicated. These equations are completed with the models for the fluid thermodynamic properties and the equation of state employed. Additional limitations on the physical model are imposed by these auxiliary equations.

Viscous Flow: The Navier-Stokes equations include the viscous and heat conduction properties of the fluid and require information on the transport property coefficients for the shear viscosity, bulk viscosity, and the thermal conductivity. These equations have the same limitations as the inviscid equations, in addition to limitations of the models for the transport properties. The Navier-Stokes equations can be used to determine the flow structure in weak shock waves. Convincing agreement with experimental data has been obtained for low Mach numbers. ${ }^{13}$ The Navier-Stokes equations for a liquid with no-slip boundary conditions can be used to determine the flow in small channels of height larger than approximately ten molecular diameters. ${ }^{14}$ For gases at low density, slip and temperature jump boundary conditions are required. At lower densities, the continuum approach becomes unsalvageable.

Gas with Vibrationally Excited Molecules: For gases at elevated temperatures the vibrational energy levels of the molecules become excited and this results in the specific heats 
of the gas becoming a function of temperature. For air, this effect becomes important at temperatures above about $700 \mathrm{~K}$. This additional physics requires a vibrational rate equation if nonequilibrium effects are important or at high gas densities an equilibrium assumption can be used. For flow in a hypersonic wind tunnel nozzle, this additional physics is required for accurate flow predictions. A good example of this type of model is given by Canupp et al. 15

Inert Gas Mixtures: The governing equations for a mixture of perfect gases are developed from the theory of gas kinetics 12 and these equations are well known. The gas mixture equations are nearly the same as the Navier-Stokes equations except a conservation of species equation is added for each species, the energy equation has an additional term due to diffusion of species, and the viscosity and thermal conductivity transport properties of the mixture are required. In addition, the diffusion velocity of the species is required. The complete theory accounts for diffusion due to concentration, temperature and pressure gradient in the flow. Because this theory is rather complex and computationally expensive, most CFD codes use some approximations, such as; binary diffusion coefficients being used rather than multicomponent diffusion coefficients; thermal and pressure diffusion being neglected; or approximate mixture rules for determining the viscosity and thermal conductivity of the gas mixture being employed. Significant errors can occur if the appropriate physics are not included; for example, expansion of gas mixtures in a rarefied jet ${ }^{16}$ requires that pressure diffusion effects be included in the diffusion model.

Chemically Reacting Gas: For gas mixtures with chemical reactions, the inert gas mixture equations are appropriate when a production term is added to the conservation of species equations. The production terms are readily determined after the chemical model has been specified. The chemical model requires that the chemical species be determined and the chemical reactions must be identified. For each chemical reaction used in the model, the forward and backward reaction rates must be known. For air, a reasonable chemical model has been determined while for other gas mixtures the chemical models range from well established to poorly known. The chemically reacting gas model is required for air when the gas temperature is greater than approximately $2000 \mathrm{~K}$ and the density is sufficiently low that the reaction rate times are less than the characteristic flow times. At higher densities the chemical equilibrium assumption can be employed and the conservation of species equations are replaced with conservation equations for the chemical elements if the element composition of the mixture varies spatially.

Transitional/Turbulent Flow: The previous flow models have assumed the Reynolds number is sufficiently low that the flow is laminar. The theory for predicting when the flow transitions to turbulent flow is an area of considerable fluid dynamics research. However, even reliable engineering techniques for predicting transition are presently lacking. A new approach to simulate transition introduces additional modeling to predict the stability of the flow using the Parabolized Stability Equations. ${ }^{17}$ This technique requires the solution of additional PDE's and has the potential of providing a technique to predict the beginning of transition.

A large number of turbulence models have been developed, with the greatest effort devoted to the incompressible and low speed flow cases. When averaging methods are utilized, such as Reynolds averaging, the governing equations will have additional terms introduced. In the conservation of momentum equation, Reynolds stress terms are added. In the conservation of 
energy equation, a new term results from the diffusion of the total energy due to turbulent motion. In the conservation of species equation, a new term results from the turbulent mass transfer. The modeling of these additional terms is performed at different levels of approximation, which can require algebraic expressions in their simplest form, and solutions of additional partial differential equations in their most complex form. For turbulent reacting flows, Probability Density Function methods, or similar techniques, have been developed but are not always sufficiently accurate. The evaluation of accuracy and limitations of the various models is an ongoing activity of the turbulence modeling community. Turbulence modeling is the single most important limitation to obtaining accurate simulations to many flows of engineering interest.

Additional Physical Phenomena: There are additional physical phenomena of increasing complexity that can be included in flow models, such as; thermal nonequilibrium, ionized flows, radiative energy transfer in gases, and multi-phase mixtures. These areas are not addressed because they introduce significantly increased levels of complexity in their own right. When these elements of physics are coupled with the previously discussed fluid physics, one ventures beyond the scope of this article. This is not to say that CFD simulations in these coupled fluid physics areas are futile. We simply recognize that the physical model unknowns and errors in these numerical simulations can dominate the discussion instead of complementing it. Theoretical derivation of the governing equations for many complex flow phenomena has not been adequately developed as yet.

Temporal and Spatial Characteristics: Beyond the fluid physics modeling issues, there are two other key areas characterizing the partial differential equations; temporal nature and spatial dimensionality. Most CFD practitioners think of these areas as unrelated, but recent work is pointing out that they are more closely related than generally thought. ${ }^{18-22}$ It is common for a CFD analyst to presume that a steady-state solution exits for a given physical model. This assumption is separate from the issue that a steady-state solution is known not to exist for a model, but one hopes that one exists for the discrete equations. It is well known that above a critical Reynolds number most steady flows become unsteady, but remain laminar up to some higher Reynolds number.

A classical flow that demonstrates both unsteady and steady behavior is low Reynolds number flow perpendicular to a long circular cylinder. For Reynolds numbers less than 49 the flow has been shown experimentally to be steady and two-dimensional. ${ }^{18,19}$ For Reynolds numbers between 49 and 180, only two-dimensional, unsteady flow exists. For Reynolds numbers above 180 , it is believed that only three-dimensional, unsteady flow exists. Recent computational work by Mittal and Balachandar 20 has shed light on this issue. They computed the flow at a Reynolds number of 525 using both a two-dimensional and a three-dimensional simulation. They found that both solutions converged to a periodic solution, but the mean drag coefficient for the 2-D simulation was 1.44 while the 3-D simulation produced a value of 1.24. Experimental measurements yield a value very near their 3-D simulation value. The point of this example is three fold. First, a 2-D unsteady physical modeling assumption seems to be appropriate and produces reasonable computational results, but it has no relationship to reality. Second, these feature are separate from differing convergence characteristics depending on the 
grid resolution of the discrete formulation. Third, be aware that this is a relatively simple flow compared to the complex flow physics discussed above. With the change of one parameter (Reynolds number), over a relatively small range, three fundamentally different flow fields, $i$. $e$., solutions to the Navier-Stokes equations, emerge. Other flow field examples with this character have been recently appeared in the literature. 21,22

\section{Auxiliary Physical Models}

Auxiliary physical models complete the equation set needed to describe the flow of interest. These auxiliary models may be given by a variety of equation types, from very simple algebraic equations to nonlinear partial differential equations.

Equation of State: The simplest equation of state is the relation for a perfect gas where the pressure is a function of the density, temperature, and molecular weight. The molecular weight is considered constant for this case. For air, this relation begins to become inaccurate at temperatures above about $700 \mathrm{~K}$, where vibrational excitation of the molecules begins. The perfect gas relation is valid at low pressures and becomes inaccurate at a pressure above approximately 10 atmospheres. For a mixture of ideal gases, the form of the equation of state remains the same, except the molecular weight is determined from the mass fraction and molecular weight of all of the species present. If the gas model uses the species conservation equations, then the equation of state should provide accurate results. If the gas is assumed to be in chemical equilibrium, then errors can be introduced into the equation of state if the equilibrium assumption is not satisfied.

Thermodynamic properties: The thermodynamic properties of many individual chemical species have been calculated with the theory of statistical thermodynamics from a first principles approach. The specific heat at constant pressure, enthalpy, and internal energy are usually determined as a function of temperature. These results are tabulated for a large number of species over certain temperature ranges and the results are usually considered to be accurate for chemical species that have been studied. Errors can be introduced as these properties are approximated with curve fits. A review of the thermodynamic properties of an 11-species air model has been given by Gupta, et al. ${ }^{23}$ Thermodynamic properties for gas mixtures can be determined from the species thermodynamic properties and the gas mixture composition. If the gas is assumed to be in chemical equilibrium and the element composition fixed, then the composition is required to be determined only once for a range of temperatures and densities. Curve fits of the thermodynamic properties of equilibrium air have been developed by Tannehill ${ }^{24}$ and Liu and Vinokur 25 . It was found that slight discontinuities in the Tannehill curve fits can cause numerical convergence problems. 26

Transport properties: The basic governing equations require a model for the stress tensor. For gases, the stress tensor is the Newtonian form while the physical behavior of some liquids can be more complex and require a non-Newtonian stress model. For example, for polymeric liquids the non-Newtonian constitutive equations are reviewed in the article by Bird. ${ }^{27}$ For the simplest Newtonian flow models the shear viscosity, bulk viscosity, and the thermal conductivity for the fluid are required. For a mixture of perfect monatomic gases, the bulk 
viscosity coefficient is zero and this is the usual assumption used in CFD codes. This assumption is not appropriate for acoustic motions and in the interior of shock waves. For air and a perfect gas assumption, the viscosity is usually approximated with the Sutherland law. The relation is accurate for temperatures between $100 \mathrm{~K}$ and $2000 \mathrm{~K}$. At lower temperatures, Keyes' viscosity relation should be used. 28 The thermal conductivity is typically obtained from the assumption of a constant Prandtl number and a constant specific heat. Errors in the thermal conductivity start to occur at temperatures above about $700 \mathrm{~K}$. For flows with a mixture of chemical species, the viscosity, frozen thermal conductivity, and binary diffusion coefficients are determined from the kinetic theory of gases. Curve fits for these properties for air species have been given by Gupta, et al. ${ }^{23}$ These properties are believed to be of reasonable accuracy, but an estimate of the error in these models is not available. The transport properties of the gas mixture are usually determined from approximate mixture rules, rather than the complete relation from the kinetic gas theory. For air with sublimation products of graphite, for example, Ryabov $^{29}$ has determined that the error in the mixture viscosity is less than $5 \%$, while the error in the frozen thermal conductivity is less than $10 \%$. Ryabov indicates that the Kendall approximation for binary diffusion coefficients gives errors below $11.5 \%$. The impact of these errors on flow results does not appear to have been evaluated.

Chemical model, reactions, and rates: The accuracy of chemical models is usually determined by the extent of research devoted to the particular gas model. Also, many models for a gas, or gas mixtures, are a simplification of a more complete model in order to obtain reasonable computation times. For air, sufficient knowledge is available to decide the appropriate species to include and what reactions are necessary. There are generally large errors in the reaction rates for the various chemical reactions, but several models have evolved as standards for air. 30 The accuracy of the simpler chemical models decreases at higher temperatures, however. When the flow of a new gas or gas mixture is being calculated, the chemical model needs careful evaluation to determine if accurate results are being obtained.

Turbulence model: The accuracy of turbulence models must be determined with experimental results for a wide range of Reynolds number, Mach number, flow and shear layer geometries, pressure gradients, and possibly other flow physics, such as combustion and multiphase flow. Recently, direct numerical simulation has provided useful information for model evaluation, complimenting experimental data. The direct numerical simulation results have been limited to low turbulence Reynolds number flows and to very simple flow geometries. The evaluation of turbulence models requires a variety of experiments which test the ability of the model to simulate turbulent flows of increasing flow complexity. Presently, turbulence models can be best evaluated with the benchmark test cases developed at the Stanford Conferences on turbulent flows. ${ }^{31}$ These experiments have been carefully picked as the best available, as far as their accuracy and specification of flow conditions required to perform numerical simulations. Also, a variety of experiments are required to test gas models for different levels of complexity; for example, incompressible flow, compressible flow, hypersonic flow, multi-component gas mixtures, reacting gas mixtures, two-phase flow, etc. Many codes use wall functions to remove the requirement of a fine grid near walls needed to resolve rapidly varying turbulence model quantities. The wall function approach can reduce the required number of mesh cells by a factor of two. For some flows, for example, near boundary 
layer separation and in separated regions, the velocity profile near a wall is not adequately represented with a logarithmic region. For these case, the wall function approach can introduce significant errors and is not reliable for skin friction and heat transfer predictions.

\section{Boundary Conditions for the Partial Differential Equations}

The boundary conditions required for the solution of the governing partial differential equations and the auxiliary equations have received limited attention in fluid dynamics. As pointed out by Oliger and Sundstrom ${ }^{32}$ nearly twenty years ago, discretization of the boundary conditions has been studied before the appropriate boundary conditions have been established for the partial differential equations. Only after difficulties with computational boundary conditions have arisen, has there been interest in understanding the appropriate boundary conditions that should be used. The article by Oliger and Sundstrom is one of the few investigations that treat determination of the boundary conditions for fluid dynamics equations (mostly inviscid flows) to ensure a well-posed problem. As these authors point out, one cannot expect reasonable numerical solutions unless the correct PDE boundary conditions have been approximated. It seems this lack of attention to what are the correct boundary conditions for the partial differential equations has worsened in the last twenty years. Recently, however, the tide seems to be turning 33-36.

There are three types of boundaries that occur: wall boundaries, open boundaries, and free surfaces. The various conditions specified at these boundaries will now be discussed.

Wall Boundary Conditions: These conditions generally have clear physical significance and the appropriate boundary conditions are easier to determine. For example, for continuum flow at a rigid, non-porous wall, one commonly has the simple conditions; velocity components are zero at the wall and the fluid adjacent to the wall is at a specified temperature, i. e., the known wall temperature. A slightly more complex boundary condition is a statement of a normal gradient to the wall. This type of boundary condition requires information from the flow solution, i. e., the interior of the solution domain. What additional condition must be added: the normal momentum equation or the zero pressure gradient normal to the wall? For near noncontinuum flows, there is velocity slip and temperature jump at a wall that must be modeled. It is more difficult to specify the appropriate wall boundary conditions for the chemical species equations because the heterogeneous chemical reactions of the gas species at the wall must be modeled. Some related open questions are:

- What are the proper turbulence conditions for a porous wall?

- At what length scale do the individual jets of a porous wall have to be modeled?

- At what length scale should the irregularities of a rough wall be modeled in a Reynolds Averaged Navier-Stokes formulation?

-What are the appropriate wall boundary conditions when vibrational nonequilibrium effects are included in the gas model with a separate vibrational temperature? 
Two additional sources of error related to wall boundary conditions are inaccurate representation of the wall geometry, and boundary discontinuities, or mathematical singularities. Inaccurate wall geometry refers to the difference between the actual physical geometry and its computational representation, i.e., fidelity of the computational to the physical geometry. Examples of these are the following: physical hardware not accurately fabricated to specifications; inaccurate CAD/CAM representation of a surface; and lack of surface roughness specification for a turbulent flow simulation. Discontinuities in the boundary conditions occur, for example, where a wall can change from solid to porous with a step change in the surface normal velocity. There is also a discontinuity at the intersection of a sliding wall and a fixed wall; for example, the classic driven-cavity problem. Another example is the singularity at the stagnation point on an axisymmetric blunt body in a cylindrical coordinate system. For the PDEs these singularities can usually be dealt with, such as with one sided limits and integrals, because of continuum mathematics. For discrete mathematics, however, singularities and discontinuities present very difficult numerical approximation issues.

Open Boundary Conditions: These are conditions that are specified along a boundary, or portion of a boundary, where there is "free" (i. e., unrestricted) inflow and/or outflow. We describe this type of boundary as free, simply to distinguish it from inflow or outflow through a porous wall discussed above. These types of boundary conditions typically result from a requirement that numerical solutions be obtained over a limited region of the flow domain. In this sense, errors associated with these boundaries could be considered to be associated with boundary conditions for the discretized formulation. However, this is not always the case. If one were simulating the flow over a model in a wind tunnel, then the exact inflow boundary conditions must be given by a specification of appropriate dependent variables in the partial differential equations and auxiliary equations at, say, the beginning of the test section. We say appropriate because specifying all dependent variables will cause an inconsistency with the PDEs and the auxiliary equations. The dependent variables would have to be measured spatially and possibly temporally if, for example, turbulence quantities were needed. This level of detailed knowledge, or calibration, of wind tunnels does not presently exist. It has also been found that specified flow quantities are commonly not consistent with the partial differential equations on the interior of the solution domain and, as a result, rapid changes occur in variables downstream from the inflow boundary.

It was recently stated by Sani and Gresho, 34 and we fully agree, that boundary conditions at open boundaries are often the most difficult and elusive aspect of mathematical modeling. The number of physical boundary conditions required, and allowed, at an open boundary is determined from an evaluation of the characteristics of the governing partial differential equations. The number of physical boundary conditions must be the same as the number of characteristics of the governing equations entering the computational domain. If the flow is locally supersonic, there is no upstream influence and accurate boundary conditions can be specified without much difficulty. Inflow, more specifically upstream, boundary conditions can typically be much closer to the region of interest than outflow, or downstream, boundary conditions. For subsonic flow over an isolated body, the boundary conditions should be applied at infinity where the flow is uniform and known. If the downstream location of the computational boundary is not located sufficiently far downstream, some flow may be re- 
entering the computational domain. Re-entrant flow boundary conditions may be expedient for obtaining solutions, but we have not seen any approaches mathematically justified for PDEs or for difference equations. More will be said on this topic when discretization errors are discussed.

Free Surface Boundary Conditions: This case is more general and far more complex than the wall case. The development of the appropriate physical relations has been presented by Batchelor. ${ }^{37}$ The general approach considers the relations that must be satisfied at the boundary between two media. At this interface the temperature of the two materials is the same and the heat flux normal to the boundary is equal on both sides. A balance of stress at the boundary with the effect of surface tension taken into account results in two transition relations. In addition, the normal component of velocity is continuous across a material boundary separating a fluid and another media and there can be mass transport at the boundary. The boundary condition relations require a value of the surface tension for the materials involved. Also, these boundary conditions can become quite difficult to apply if the interface becomes unstable with large movement. For example, it is well known that the RayleighTaylor instability occurs when a heavy fluid is above a light fluid in a gravitational field.

\section{Discretization and Solution Errors}

Discretization errors are those caused by the numerical replacement, or discrete mapping, of the partial differential equations, the auxiliary physical models, and the continuum boundary conditions into algebraic equations. Solution errors refer to the errors in the computer solution of the complete set of the discrete, i. e., algebraic, equations. Errors generated by or associated with each of these sub-categories is now discussed.

\section{Discretization of the Partial Differential Equations}

The reformulation of the partial differential equations into algebraic equations that accurately represent the original equations is a bigger, more problematic, mathematical step than is generally recognized. The formal mathematical theorem relating the differential and difference equations is known as Lax's Equivalence Theorem (LET). 38-40 The theorem is built upon two requirements of the discrete method; consistency and stability of the method. A discrete method is consistent if it can be analytically shown that as the discretization size approaches zero, the difference equations are equal to the differential equations. This can be written as

$$
\text { Consistency Definition: } \lim _{\Delta \rightarrow 0} \delta_{\Delta} \Phi=\partial \Phi
$$

where $\delta_{\Delta} \Phi$ represents the system of finite difference equations (which includes any method for discretizing the partial differential equations), $\partial \Phi$ represents the original system of partial differential equations of the mathematical model, and $\Delta$ represents the size of all discretized independent variables. A numerical method is stable if it can be analytically shown that as the 
solution is marched in time, or is iteratively solved, the solution remains bounded. One can write this as

$$
\text { Stability Definition: } \lim _{\mathrm{n} \rightarrow \infty}\left\|\Phi_{\Delta}^{\mathrm{n}}-\Phi_{\Delta}^{0}\right\|<\mathrm{N}
$$

where $\Phi_{\Delta}^{\mathrm{n}}$ represents all dependent variables of the system of equations at time $\mathrm{n}$ for a fixed mesh size $\Delta, \Phi_{\Delta}^{0}$ represents arbitrary initial values of the dependent variables, and $N$ is an arbitrary constant.

It can be proven mathematically that the above conditions are necessary and sufficient for the solution of the discretized system of equations to be identical to that of the partial differential equations, for linear problems. This proof is the foundation of the numerical solution of partial differential equations. The fact that LET provides necessary and sufficient conditions only for linear PDEs is not widely recognized. There seems to be some understandable reasons for this. In Richtmyer and Morton's classical text, 38 the qualification to linear problems was not mentioned. Some modern texts 39,40 clearly make the point that the theorem applies to linear problems. For nonlinear problems, consistency and stability of the numerical method are necessary, but not sufficient, for convergence of the numerical solution to the continuum solution. Although the mathematical bedrock of numerical solutions is much thinner than generally believed, the difficulties and hindrances to progress in CFD also stem from other sources. Some difficulties are practical in nature and some are implementation issues. In the following we discuss some of these issues.

Approximate Consistency and Stability Analyses: Consistency and stability proofs of numerical methods are predominantly developed for very simple model problems, never on a "real" nonlinear problem. The model equations are always linearized equations and uncoupled from any other equations. For example, the most commonly used models are one-dimensional versions of the wave equation, heat conduction equation, and viscous Burger's equation. These simple, linearized, uncoupled, one dimensional equations do not exhibit the astounding spectrum of solutions exhibited by the Navier-Stokes equations (vortices cannot exist in onedimension, for example). If additional complex physics such as gas mixtures, turbulence, and reacting flow are included, it is clearly seen that these model equations are far removed from real world problems. The reason for the elimination of these real world complexities, of course, is that the difference equations resulting from the analysis are nonlinear, just as the original partial differential equations, and cannot be completely analyzed mathematically.

Additional, but related, simplifications of consistency and stability analyses are elimination of: mixed classification partial differential equations, non-uniform grids, and boundary and initial conditions. Stability analyses are never done on difference equations with mixed classification, e. g., hyperbolic and elliptic. These mixed zones, however, very commonly exist. For example, in every non-trivial supersonic flow problem modeled by the steady-state Navier-Stokes equations, hyperbolic and elliptic regions exist adjacent to one another. In the extremely rare event that a multi-dimensional stability analysis is conducted, a structured grid is always assumed to be uniformly spaced. This assumption does not correspond to real world problems. Boundary condition type and geometry can influence the stability of numerical 
methods. Only those analyses that include the discrete boundary conditions, along with the discrete linearized equations, can provide the correct behavior of the numerical scheme. 40

LET In Practice: The matching of solutions with difference and differential equations using consistent and stable numerical methods applies only in the limit as the mesh and time step approach zero. The reality, of course, is that all numerical solutions are obtained for finite grids and time steps. Although this fact is obvious, it bears keeping in mind that the proof is a theoretical construct. In practice, equality is never attained. Examples of the mismatch between the two mathematical models are in order. The zone of influence of hyperbolic difference equations can be very different from that of the differential equations. Consider twodimensional inviscid supersonic flow. In CFD solutions, the zone of influence of the difference equations is always larger than that of the differential equations. This is because the zone of influence in the CFD solution also depends on the difference method and the grid size. This mismatch can have varying effects in the discrete solution. Note that this mismatch is separate from a CFL condition on the time-like coordinate. As a second example, it has been found by Yee et al $41-42$ that finite difference solutions can exhibit a much wider range of dynamical behaviour than their continuum counterparts. They have found that "the use of linearized analysis as a guide to studying strongly nonlinear PDEs is insufficient and can lead to misleading results." And finally, "In particular, when one tries to stretch the maximum limit of the linearized allowable time step for highly coupled...nonlinear systems, most likely all of the different types of spurious asymptotes (e.g. spurious steady states, periodic orbits, limit cycles, or chaotic phenomena) can be achieved in practice depending on the initial conditions."

A related numerical phenomena occurs in flow over slender bodies at a high angle of attack. For this flow field, vortex patterns on the leeside of the body can be symmetric or asymmetric depending on the angle of attack and freestream conditions. How the difference equations are written and solved can also determine what flow field is obtained. Levy, Hesselin, and Degani ${ }^{43}$ showed that the manner in which the left side of each difference equation is formulated in the solution procedure impacts whether the steady-state result is symmetric or asymmetric. Their studies indicate that the steady-state solution is not unique and there are at least two solutions that can satisfy the right side of the difference equations. If the difference form of the left side is not symmetric, spurious asymmetric results can be computed.

Spatial and Temporal Resolution: This category of error is the most well recognized of the discretization errors and is unquestionably the most important. This error, also referred to as truncation error, is due to finite resolution in the spatial and temporal discretization. In the past, finite difference analyses rarely attempted to estimate the magnitude of this error on the results computed. Finite element methods seem to have a better record of addressing this issue. A strong initiative by individuals such as P. J. Roache, K. N. Ghia, F. M. White, C. J. Freitas and others has raised the importance of addressing grid convergence in CFD solutions. The enforced discipline through organizations such as the ASME and the AIAA and its publications was desperately needed for the maturation of numerical simulations. Because of the importance of this topic and because effective measures can be taken to control it, this matter will be addressed in in Section V: Detection and Estimation of Errors. 


\section{Discretization of the Auxiliary Physical Models}

If the auxiliary physical model equations are linear algebraic expressions, which can be solved directly, then the errors are of the magnitude of the computer round-off and are generally very small. An example is the equation of state for a perfect gas. If the algebraic expressions are nonlinear in the unknowns, then some linearization technique, typically an iterative technique, is required for solution (note that by the term "algebraic" we also include transcendental equations). Errors can occur if the iteration is not sufficiently converged on each occasion it is used; convergence error will be discussed later. An example of a nonlinear auxiliary equation is the determination of the equilibrium chemical composition for a mixture of chemical species. Thermodynamic and transport properties for real gases are approximated with table look-up/interpolation or curve fits. The required accuracy of the approximations to these properties has not been established and, surprisingly, for many cases in the past, the approaches resulted in large errors. Clearly these errors can be reduced by more accurate interpolation techniques, but the data storage requirements are significantly increased with probable increases in computer time. The errors in the approximation of the individual properties of the chemical species must be compatible with the errors being introduced in the techniques used to approximate the gas mixture properties. Careful evaluation of accuracies required for thermodynamic and transport properties used in flow simulations and the impact on fluid dynamic predictions have not been performed.

Probably the most important auxiliary model used in CFD is the turbulence model; yet accurate models for a wide range of conditions are not available. For algebraic models, the primary error source is the determination of some flow field feature needed in the calculation, e. $g$., the magnitude of a turbulent length scale. The reliable determination of the appropriate length scale has proven to be difficult for a wide variety of shear layer geometries. By far the most difficult and computationally intensive, however, is the calculation of turbulent transport variables using two-equation turbulence models, or Reynolds stress models. The discretization error of these type of PDEs has been discussed above, and will be addressed later.

\section{Discretization and Implementation of Boundary Conditions}

The boundary conditions for the difference equations, whether they are for wall, open, or free surfaces, must provide independent, but yet consistent, information for the solution of the PDEs. Over-specification of the discrete boundary conditions (BCs) can cause divergence of the iterative, or temporal, solution. Under-specification of the BCs will cause the solution not to converge, i. e., wander about, or to converge to different solutions depending on arbitrary features, such as initial conditions, grid size, relaxation parameters, etc. This perfect balance between over- and under-specification of knowledge on boundaries is much more difficult to obtain for difference equations than it is for PDEs. We do not fully understand why this balance is more precarious for discrete equations, but we will suggest some conceptual reasons. In continuum mathematics, the PDEs are fully coupled to the boundaries. No discrepancy, inconsistency, or mismatch is tolerated for the existence of a solution. In difference equations, however, the coupling is weaker and it depends on a variety of numerical parameters, such as the iterative algorithm, spatial differencing scheme, grid size, and numerical smoothing parameters. Recent work in numerical boundary conditions supports our 
explanation. 34,44

Implementation of Dirichlet boundary conditions are usually straightforward. Neumann and mixed boundary conditions require that a difference method be used at the boundary. This method is usually of the same order accuracy as the spatial differencing method so that a globally consistent order is obtained for the scheme. However, only a grid refinement study will establish that the overall order of the complete numerical scheme is as expected. It should be noted that very few researchers have demonstrated the overall, or global, order of their simulation.

The most difficult type of boundary condition to implement is the open BC. The entire issue of inflow and outflow boundary conditions in now being studied in more depth than in the past. An excellent summary and, we believe, required reading on the subject of open boundary conditions for incompressible Navier-Stokes equations is given by Sani and Gresho. ${ }^{34}$ This article clearly shows that there are more questions than answers on the implementation of open boundary conditions, even for the relatively simple physics of steady, incompressible laminar flow. Concerning open boundary conditions, they state, "Nature is usually silent, or in fact perverse, in not communicating the appropriate ones." Also, "The boundary conditions on such open portions of the boundary are a necessary evil ... We believe that there are no 'true' open boundary conditions, thus explaining Nature's silence. We also believe and may demonstrate herein that perhaps nowhere else do theory and practice seem to clash so much." Sani and Gresho also introduced the term "fuzzy boundary conditions" to suggest the existence of numerical BCs that produce good numerical solutions, but if one takes the analytical limit as $\Delta \rightarrow 0$ of these BCs, one obtains unacceptable/incompatible BCs for the PDEs.

The vast majority of effort in the formulation and development of numerical boundary conditions has been directed toward steady flow conditions. As CFD matures, the need for reliable and accurate unsteady boundary conditions will become more important. Additionally, when dealing with more complex flow physics, such as chemically reacting flows, multi-phase flows, and flows with radiative energy transport, accurate numerical boundary conditions will prove much more difficult.

\section{Solution of the Discrete Equations}

The error in the computer solution of the discrete model equations is referred to as the discrete solution error; sometimes this is referred to as the iterative convergence error and it can occur for either steady-state or unsteady simulations. This error is defined as the difference between the exact solution to the discrete equations and the approximate, i. e., computer, solution obtained. The round-off error due to the finite precision of the the computer could also be included here, but it is commonly thought of as a separate category.

Often one reads, for example, that the global iteration toward a steady state solution was performed until there was a small change in the variables between the iterations. This type of test will not always produce accurate results because the magnitude of the change between 
iterations depends on the time step, or relaxation parameter, and also on the present convergence rate of the solution. A more accurate and reliable approach is to write the difference equations in delta form and then compute the residual (right hand side of the equation) for each equation in the system at each point in the grid. At the start of the iteration, the dependent variables do not satisfy the difference equation and the residual is non-zero over much of the grid. As the iteration proceeds, the residual for all difference equations and at all spatial locations is driven to machine zero. Although this level of solution accuracy is computationally intensive and probably not required for all simulations, it is highly recommended that this iterative convergence be demonstrated on coarse grid solutions. Another approach has been suggested by Ferziger and Peric' 45 which estimates the solution convergence as the iterative process is performed.

For transient flow simulations, at each time step the difference equations must be iterated to convergence for implicit schemes so that the iterative error is much smaller than the temporal discretization error. On finite volume schemes, for example, conservation of mass, momentum, and energy can be violated with inadequate iterative convergence at each time step. When the temporal and spatial step sizes are decreased, the iterative solution at each time step could require more iterations be performed to obtain smaller iterative errors. Some numerical schemes can require no iterations when an alternating direction implicit scheme is used, while many iterations are required when a Jacobi iterative solution is used. The solution technique used for the sparse matrix solver determines the iterations required. The iterative behavior at each time step also depends on how the governing equations are solved. For example, with a segregated approach, each dependent variable is solved from a separate difference equation and is solved one at a time sequentially. With a coupled approach, all dependent variables are solved for simultaneously from all of the difference equations. The behavior of the iterative solution technique at each time step must be understood so that no significant iterative convergence errors are introduced into the solution procedure.

\section{Detection and Estimation of Errors}

The following section suggests methods for detecting and quantifying certain types of discretization errors. Some of these methods are similar to the types of error detection and control methods incorporated in modern software packages for solving ordinary differential equations. Modern ODE solvers are extraordinarily adaptive at controlling accuracy, maintaining numerical stability, and are very robust; features that are clearly lacking in most CFD codes.

\section{Discretization Errors}

Analytical solutions to the PDEs of interest provide an extremely valuable tool in demonstrating code verification. At the present time, use of analytical solutions in code verification seems to be distinctly out of favor in the CFD community. There are a surprisingly large number of exact and approximate, but highly accurate, analytical solutions to special cases of the Euler and Navier-Stokes equations ${ }^{46-50}$. Possibly the reason for lack of interest 
and familiarity with analytical solutions among many CFD code developers is the lack of exposure to these methods in modern graduate education.

During the last several years a novel approach has been used to obtain exact solutions to equations very similar to the Navier-Stokes equations. In the approach developed by Steinberg and Roache, 51 a specific form of solution function is assumed to satisfy the PDE of interest. This function is inserted into the PDE and all the derivatives are analytically computed using symbolic manipulation software, such as MACSYMA ${ }^{\mathrm{TM}}$. The equation is simplified, and all remaining terms resulting in inequality between both sides of the equation are grouped into a forcing function term. This term is then considered to be simply added to the original PDE so that the assumed solution function satisfies the new PDE exactly. For example, in the Euler or Navier-Stokes equations this term can be considered to be a source term. The boundary conditions for the new PDE are considered to be either the value of the solution function on the boundary (Dirichlet condition), or a Neumann condition that can be analytically derived from the solution function. A good description for these constructed analytical solutions is manufactured solutions.

To verify the CFD code using this technique, the computed source term and boundary conditions are programmed into the code and a numerical solution computed. This procedure verifies, albeit for a narrow range of physical modeling, a large number of numerical aspects in the code, for example; the numerical method, differencing technique, spatial transformation technique for grid generation, grid spacing technique, and coding correctness. Shih, Tan and Hwang 52 have taken this approach a step further and applied it to the incompressible NavierStokes equations for laminar, two-dimensional flow. They have obtained an impressive exact solution to the classical lid-driven cavity problem for an arbitrary Reynolds number. It is highly recommended that incompressible Navier-Stokes codes be verified with this exact solution.

\section{Grid Resolution Errors}

The largest contributor to numerical solution error, and the one that has caused the most inaccuracy in CFD solutions, is the one caused by inadequate grid resolution. It is ironic that the quantification of this error is also the most straightforward. We believe the reason for this paradox is simple: cost. The grid size used for a numerical solution is usually at the limit of computer time or budgetary constraints; sometimes the grid used is simply considered "good enough" for the simulation at hand. The computational cost, and to a lesser extent personnel time cost, to carefully conduct a grid sensitivity study is roughly a factor of 4 larger for 2-D problems and a factor of 8 larger for 3-D problems beyond an "acceptable" solution. Consequently, grid resolution studies were rarely done until recently. We submit that our blunt assessment of the situation is supported by simply examining the state-of-the-art in ODE solvers. ODE solvers suffered the same paradox during their early years, but they are now the shining example for accuracy and reliability for numerical solutions. The reason they have progressed so rapidly in this regard is because the computer power required for their solution is usually minimal compared to the solution of PDEs.

For finite difference methods, spatial discretization error is estimated using Richardson's 
classical method, also known as "deferred approach to the limit" and "iterated extrapolation." 53 Using Richardson's extrapolation, one can write

$$
\Phi_{\text {exact }}=\Phi_{\Delta}+\alpha \Delta^{\mathrm{p}}+\text { higher order terms in } \Delta
$$

where $\Phi_{\text {exact }}$ is the exact solution to the PDE, $\Phi_{\Delta}$ is the numerical solution obtained using a grid size of $\Delta, \alpha$ is a constant for a given numerical solution, and $\mathrm{p}$ is the order of accuracy of the numerical method. It is important to note that Richardson's method applies not only to computed dependent variables at all grid points, but also to solution functionals. Solution functionals are integrated and differentiated quantities such as body lift and surface heat flux, respectively. If $\mathrm{p}$ is known, then two numerical solutions with different grid sizes are required to compute $\Phi_{\text {exact }}$ and $\alpha$ so that the discretization error can be estimated. The fine grid solution need not be twice the number of grid points (in each coordinate direction) as the coarse grid, but this is common practice and it provides a more accurate estimate. Roache ${ }^{54}$ has developed a Grid Convergence Index (GCI), based on Richardson's extrapolation, that converts error estimates obtained from any grid refinement ratio into an equivalent grid doubling estimate. He argues that using the GCI would help standardize the accuracy evaluation of grid refined solutions.

Careful use and accurate estimation of error using Richardson's method has been documented only by a few researchers (see, for example, Refs. 11, 55-58). In most cases it has been found that two solutions are insufficient to properly use Richardson's method. The reason three or more solutions are required is that from the first two solutions it may be found that the demonstrated order of accuracy from the calculations does not match the formal, or expected, accuracy of the method. This result can be caused by one of two reasons. First, numerical inaccuracies or errors, such as excessive grid stretching, inaccurate implementation of boundary conditions, and coding errors, cause a degradation in the global accuracy of the method. Second, it is commonly found that insufficient grid resolution was used on the first two solutions such that formal accuracy is not attained until finer grids are used. Until the computed grid convergence rate from two individual solutions, with the same grid clustering, matches the known (or previously demonstrated) order of accuracy of the code, Richardson's method cannot be used to estimate error. In addition, the accuracy must be demonstrated for the parameters of interest, either local or global, and not simply be implied from other parameters.

A final observation is made that apparently has not been pointed out in the literature concerning the use of Richardson's method in error estimation. By examining the grid convergence plots of the researchers that have carefully used Richardson's method an interesting, but unsettling, feature is observed. Figure 1 shows a grid convergence plot for a steady-state, three-dimensional, Navier-Stokes solution taken from Walker and Oberkampf. 57 The convergence history is for a high Reynolds number, laminar, perfect gas, hypersonic flow with a highly clustered grid in the boundary layer. The geometry is spherically blunted cone with a slice on one side of the body at an angle of attack of $16^{\circ}$. The aerodynamic normal force coefficient, $\mathrm{C}_{\mathrm{N}}$, pitch moment coefficient, $\mathrm{C}_{\mathrm{m}}$, and the axial force coefficient, $\mathrm{C}_{\mathrm{A}}$, are each shown for the conical and sliced portions of the body. The spatial differencing method is 
formally second order accurate, and has been demonstrated to be such in previous verification computations, in the circumferential direction. It is seen that the grid fineness required to obtain second order of accuracy of the code is also a grid that produces relative errors on the order of $0.1 \%$. In other words, to demonstrate grid convergence using Richardson's method one must compute solutions with very fine grids that have relative errors on the order of $0.1 \%$. We suggest that the reason for this character is the large gradients in the boundary layer, i. e., the nonlinear feature of the equations. If less accurate and less expensive solutions, say $10 \%$ error, are desired in production CFD solutions, we have seen no evidence that Richarson's method is usable for moderate grid resolution in high Reynolds number flows.

\section{Conclusions}

In this article we draw a sharp distinction between physical modeling issues and numerical formulation and solution issues. This perspective is rarely taken; in fact, the clear trend in large scale numerical simulations is to homogenize the two issues. We believe additional merging of these issues will lead to increased confusion as to the cause of inaccuracy and, as a result, loss in confidence in numerical simulations. To achieve the level of maturity in CFD characterized by "value exceeds expectation" and "most analysis done without supporting experimental comparisons," will require a much deeper understanding of fluid physics, its mathematical modeling, and numerics; and their relationships.

Computational fluid dynamics, as other computational nonlinear physics, is still in an early stage of development and application. The progress in CFD during the last thirty years is most impressive. This progress has resulted because of advances in each contributing area; physical modeling, numerical algorithms, grid generation, and computer speed. Highly accurate simulations, however, have only been demonstrated for relatively simple problems in fluid mechanics, problems that have only one type of nonlinear feature. Examples of these are steady incompressible laminar flow over a backstep and steady inviscid supersonic flow over a blunt body. When viewing the vast and dazzling array of complexity of fluid mechanics in nature, we must conclude that a great deal of caution must be exercised in claiming confidence in numerical simulations of processes involving multiple nonlinear physics that are highly coupled. 


\section{References}

1. Bradley, R. G., Current Capabilities and Future Directions in Computational Fluid Dynamics, National Research Council, National Academy Press, Washington, DC, 1986.

2. IEEE Standard Dictionary of Electrical and Electronics Terms, F. Jay, editor, Institute of Electrical and Electronics Engineers, ANSI/IEEE Std. 100-1984.

3. American National Standard Guidelines for the Verification and Validation of Scientific and Engineering Computer Programs for the Nuclear Industry, American Nuclear Society, La Grange Park, IL, ANSL/ANS-10.4-1987, 1987.

4. Bradley, R. G., "CFD Validation Philosophy," AGARD Symposium on Validation of Computational Fluid Dynamics, Conf. Paper No. 1, Lisbon, Portugal, 1988.

5. International Organization for Standardization, "Quality management and quality assurance standards--Part 3: Guidelines for the application of ISO 9001 to the development, supply and maintenance of software," ISO 9000-3:1991(E), 1993.

6. Mehta, U., "Some Aspects of Uncertainty in Computational Fluid Dynamics Results," J. of Fluids Engineering, Vol. 113, No. 4, 1991, pp. 519-525.

7. Mehta, U., "Guide to Credible Computer Simulations of Fluid Flows," J. for Propulsion and Power, Vol. 12, No. 4, 1996.

8. Oberkampf, W. L., "A Proposed Framework for Computational Fluid Dynamics Code Calibration/Validation,” AIAA Paper No. .94-2540, June 1994.

9. Schlesinger, S., et al, "Terminology for Model Credibility," Simulation, Vol. 32, No. 3 , Mar. 1979. pp. 103-104.

10. Boehm, B. W., Software Engineering Economics, Prentice-Hall, 1981, p. 37.

11. Blottner, F. G., "Accurate Navier-Stokes Results for the Hypersonic Flow Over a Spherical Nosetip," J. of Spacecraft and Rockets, Vol. 27, No. 2, 1990, pp. 113-122.

12. Hirschfelder, J. O., Curtiss, C. F. and Bird, R. B., Molecular Theory of Gases and Liquids, John Wiley \& Sons, Inc., New York, 1954.

13. Vincenti, W. G. and Kruger, C. H. Jr., Introduction to Physical Gas Dynamics, John Wiley \& Sons, Inc., New York, 1965, p. 412.

14. Koplik, J. and Banavar, J. R., "Continuum Deductions from Molecular Hydrodynamics", Annual Review of Fluid Mechanics, Vol. 27, 1995, pp. 257-292. 
15. Canupp, P. W., Candler, G. V., Perkins, J. N., Erickson, W. D., "Analysis of Hypersonic Nozzles Including Vibrational Nonequilibrium and Intermolecular Force Effects," AIAA Journal, Vol. 31, No. 7, 1993, pp. 1243-1249.

16. Rothe, D. E., "Electron Beam Studies of the Diffusive Separation of Helium-Argon Mixtures," Physics of Fluids, Vol. 9, Sept. 1966, pp. 1643-1658.

17. Herbert, T., "Parabolized Stability Equations," Annual Review of Fluid Mechanics, edited by J. L. Lumley, M. Van Dyke, and H. L. Reed, Vol. 29, 1997, pp. 245-283.

18. Williamson, C. H. K. and Roshko, A., "Measurements of Base Pressure in the Wake of a Cylinder at Low Reynolds Numbers Relationship for the Laminar Vortex Shedding," Z. Flugwiss. Weltraumforsch, Vol. 14, 1990, pp. 38-46.

19. Roshko, A., "Perspectives on Bluff Body Wakes," J. Wind Engr. and Industrial Aero., Vol. 49, 1993, pp. 79-100.

20. Mittal, R., and Balachandar, S., "Vortical Structures in Bluff Body Wakes," AIAA Paper No. 95-0867, Jan. 1995.

21. Pironneau, O., "Summary and Conclusions for Test Case T2," in Numerical Simulation of Unsteady Flows and Transition to Turbulence, Edited by O. Pironneau, W. Rodi, I. L. Ryhming, A. M. Savill, and T. V. Truong, Cambridge University Press, 1990.

22. Rudy, D. H., Thomas, J. L., Kumar, A.,Gnoffo, P. A., and Chakravarthy, S. R., "Computation of Laminar Hypersonic Compression-Corner Flows," AIAA Journal, Vol. 29, No. 7, July, 1991, pp. 1108-1113.

23. Gupta, R. N., Yos, J. M., Thompson, R. A. and Lee, Kam-Pui, "A Review of Reaction Rates and Thermodynamic and Transport Properties for an 11-Species Air Model for Chemical and Thermal Nonequilibrium Calculations to 30,000K," NASA Reference Publication 1232, August 1990.

24. Tannehill, J. C.. and Mugge, P. H., "Improved Curve Fits for the Thermodynamic Properties of Equilibrium Air Suitable for Numerical Computation Using Time-Dependent or Shock-Capturing Methods," Engineering Research Institute, Iowa State University, Ames, Iowa. Feb. 1974.

25. Liu, Y. and Vinokur, M.. "Equilibrium Gas Flow Computations. I. Accurate and Efficient Calculation of Equilibrium of Equilibrium Gas Properties," AIAA Paper No. 89-1736, June 1989.

26. Blottner, F. G. and Larson, D. E., "Navier-Stokes Code NS3D for Blunt Bodies Part I: Analysis, Results, and Verification," Sandia National Labs., Albuquerque, NM, SAND880504/1, March 1988. 
27. Bird, R. B., and Wiest, J. M., "Constitutive Equations for Polymeric Liquids", Annual Review of Fluid Mechanics, Vol. 27, 1995, pp. 169-193.

28. Keyes, F. G., "A Summary of Viscosity and Heat Conduction Data for He, A, H2, O2, N2, CO, CO2, H2O and Air," Trans. ASME, Vol. 73, 1951, pp. 589-596.

29. Ryabov, V. V., "Transfer Coefficient of Multicomponent Air with Sublimation Products of Graphite," J.of Engr. Phy., Vol. 55, July 1988, pp. 786-791.

30. Anderson, J. D., Jr., Hypersonic and High Temperature Gas Dynamics, McGraw-Hill, New York, 1989.

31. Bradshaw, P., Editor, "Collaborative Testing of Turbulence Models - Collected Results of an International Project," Mechanical Engr. Dept., Stanford University, Stanford, CA 94305-3030, August 1994.

32. Oliger, J. and Sundstrom, A., "Theoretical and Practical Aspects of Some Initial Boundary Value Problems in Fluid Dynamics," SIAM J. Applied Math., Vol. 35, 1978, pp. 419446.

33. Verhoff, A. and Stookesberry, D., "Second-Order, Far-Field Computational Boundary Conditions for Inviscid Duct Flow Problems," AIAA Journal, Vol. 30, No. 5, 1992, pp. 1268-1276.

34. Sani, R. L. and Gresho, P. M., "Resumé and Remarks on the Open Boundary Condition Minisymposium," Intl. J. Num. Methods in Fluids, Vol. 18, 1994, pp. 983-1008.

35. Nordstrom, J., "Accurate Solutions of the Navier-Stokes Equations Despite Unknown Outflow Boundary Data," J. of Computational Physics, Vol. 120, 1995, pp. 184-205.

36. Verhoff, A., "Far-Field Computational Boundary Conditions for Three-Dimensional External Flow Problems," AIAA Paper No. 96-0892, Jan. 1996.

37. Batchelor, G. K., Introduction to Fluid Dynamics, Cambridge University Press, Cambridge, Great Britain, 1967.

38. Richtmyer, R. D. and Morton, K. W., Difference Methods for Initial-Value Problems, Interscience Publ, New York, 1967, pp. 39-48.

39. Fletcher, C. A. J., Computational Techniques for Fluid Dynamics 1: Fundamentals and General Techniques, Springer-Verlag, Berlin, 1988, pp. 73-75.

40. Morton, K. W. and Mayers, D. F., Numerical Solution of Partial Differential Equations, Cambridge Univ. Press, Cambridge, Great Britain, 1994, pp. 131-140. 
41. Yee, H., Sweby, P. and Griffiths, D., "Dynamical Approach Study of Spurious SteadyState Numerical Solutions of Nonlinear Differential Equation: 1. The Dynamics of Time Discretization and Its Implementations for Algorithm Development in Computational Fluid Dynamics," J. Comp. Phys., Vol. 97, No. 2, 1991, pp. 249-310.

42. Yee, H. and Sweby, P. K., "Dynamical approach Study of Spurious Steady-State Numerical Solutions for Nonlinear Differentia Equations, Part II: Global Asymptotic Behavior of Time Discretizations," Intl. J. of Computational Fluid Dynamics, Vol. 4, 1995, pp. 219-283.

43. Levy, Y., Hesselink, L. and Degani, D., "Anomalous Asymmetries in Flows Generated by Algorithms that Fail to Conserve Symmetry," AIAA Journal, Vol. 33, No. 6, June, 1995, pp. 999-1007.

44. Grinstein, F. F., "Open Boundary Conditions in the Simulation of Subsonic Turbulent Shear Flows," J. Comp. Phys., Vol. 115, 1994, pp. 43-55.

45. Ferziger, J. H. and Peric', M., "Further Discussion of Numerical Error in CFD," Inter. J. for Numerical Methods in Fluids, Vol. 23, 1996, pp. 1263-1274.

46. Karamcheti, K., Principles of Ideal-Fluid Aerodynamics, Wiley, New York, 1966.

47. Panton, R. L., Incompressible Flow, Wiley, New York, 1984,.

48. Hayes, W. D. and Probstein, R. F., Hypersonic Flow Theory; Vol. 1, Inviscid Flows, Academic Press, New York, 1966.

49. Wang, C. Y., "Exact Solutions of the Steady-State Navier-Stokes Equations," Annual Review of Fluid Mechanics, Vol. 23, 1991, pp. 159-177.

50. Grenda, J. M., Schwer, D. A., and Merkle, C. L., "Use of Analytical Solutions in Validating Unsteady CFD Codes," AIAA Paper No. 96-2164, June 1996.

51. Steinberg, S. and Roache, P. T., "Symbolic Manipulation and Computational Fluid Dynamics," J. Comp. Phys., Vol. 57, No. 2., 1985, pp. 251-284.

52. Shih, T. M., Tan, C. H., and Hwang, B. C., "Effects of Grid Staggering on Numerical Schemes," Intl, J. Num. Methods in Fluids, Vol 9, 1989, pp. 193-212.

53. Richardson, L. F., "The Deferred Approach to the Limit," Trans. Royal Soc. of London, Ser. A, Vol. 226, 1927, pp. 299-361.

54. Roache, P. J., "Perspective: A Method for Uniform Reporting of Grid Refinement Studies," J. of Fluids Engineering, Vol. 116, 1994, pp. 405-413. 
55. Roache, P. J., "Scaling of High-Reynolds-Number Weakly Separated Channel Flows," in Numerical Aspects and Physical Aspects of Aerodynamic Flows, edited by T. Cebecci, Ch. 6, Springer-Verlag, NY, 1982, pp. 87-98.

56. Shirazi, S. A. and Truman, C. R., "Evaluation of Algebraic Turbulence Models for PNS Predictions of Supersonic Flow Past a Sphere-Cone," AIAA Journal, Vol. 27, No. 5, 1989, pp. 560-568.

57. Walker, M. A. and Oberkampf, W. L., "Joint Computational/Experimental Aerodynamics Research on a Hypersonic Vehicle: Part 2, Computational Results," AIAA Journal, Vol. 30, No. 8, 1992, pp. 2010-2016.

58. Celik, I. and Zhang, W. M., "Calculation of Numerical Uncertainty Using Richardson Extrapolation: Application to Some Simple Turbulent Flow Calculations," J. of Fluids Engineering, Vol. 117, 1995, pp. 439-445. 


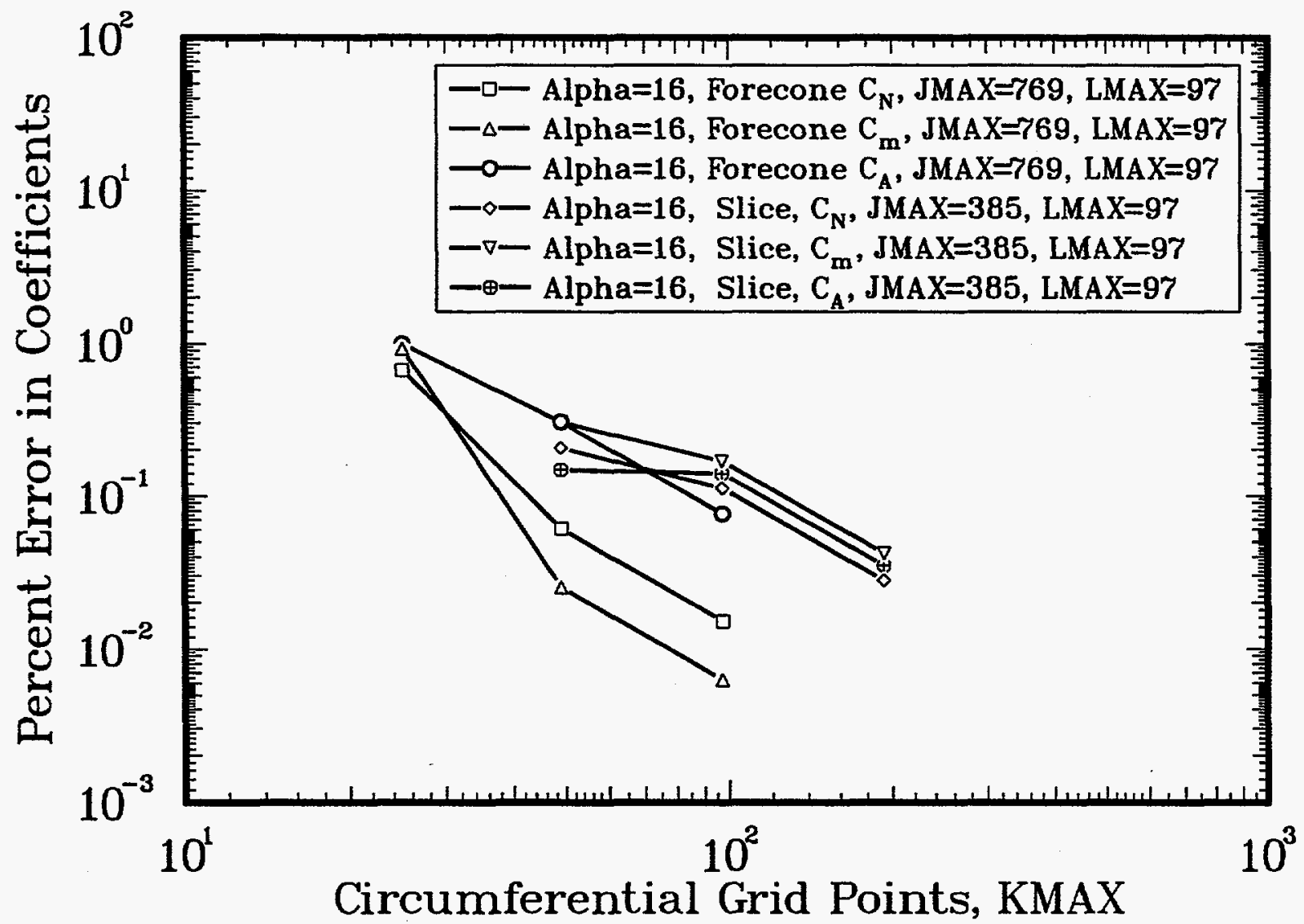

Figure 1: Grid convergence error in aerodynamic force and moment coefficients vs number of circumferential grid points for a reentry vehicle (from Ref. 57) 


\section{DISTRIBUTION:}

2 Ralph R. Jones, III

Sverdrup Tech. Inc./AEDC Group

1099 Ave. C

Arnold AFB, TN 37388-9013

2 Air Force Phillips Laboratories

Ballistic Missile Technology Program Office

VT-B

Attn: Norm Wixom

3550 Aberdeen SE

Albuquerque, NM 87117-5776

$4 \quad$ Air Force Wright Laboratories

Attn: Valentine Dahlem/FIM Jerry L. Patterson/FIMG Joseph Shang/FIM Norman Scaggs/FIMO

2645 Fifth St

Wright-Patterson AFB, OH 45433-7936

1 David Belk

WLMNAA

101 W. Eglin Blvd, Suite 219

Eglin AFB, FL 32542-6810

1 Mujeeb Malik

High Technology Corp.

28 Research Dr.

Hampton, VA 2366

2 Michael Mendenhall

Nielsen Engr. and Research, Inc.

510 Clyde Ave.

Mountain View, CA 94043

1 Robert Nelson

Dept. of Aerospace and Mechanical Engineering

University of Notre Dame

Notre Dame, IN 46556
2 John H. Fox

Sverdrup Tech. Inc./AEDC Group

740 Fourth Ave.

Arnold AFB, 37389-6001

2 NASA Lewis Research Center

Attn: Charles Towne, MS 5-11

Gary Klann, MS 5-11

21000 Brookpark Rd.

Cleveland, $\mathrm{OH} 44135$

2 Robert L. P. Voisinet

Naval Surface Warfare Center

Mail Stop K24

Dahlgren Div.

10901 New Hampshire Ave.

Silver Spring, MD 20903-5640

2 Jack Benek

Micro Craft Inc.

P. O. Box 370

207 Big Sp;rings Ave.

Tullahoma, TN 37388

2 Thomas Barber

411 Silver Lane

MS 129-20

United Technologies Center

East Hartford, CT 06108

1 Pat Roache

1108 Mesa Loop

Los Lunas, NM 87031

2 Hans Mair

Institute for Defense Analysis

Operational Evaluation Div.

1801 North Beauregard St.

Alexandria, VA 22311-1772 
$3 \quad$ NASA/Ames Research Center

Attn: Unmeel Mehta, MS T27B-2

Helen Yee, MS 202A-1

Lado Muhlstein, MS 227-4

Moffett Field, CA 94035-1000

2 Tom Harris

SAIC

997 Old Eagle School Rd

Suite 215

Wayne, PA 19087

2 T.P. Shivananda

Bldg. SB2/Rm. 1011

TRW/Ballistic Missiles Division

P. O. Box 1310

San Bemardino, CA 92402-1310

2 Steven Ashby

UNL

P. O. Box 808, L-561

Livermore, CA 94551

2 Ken Hanson

MS P940, DX-3

LANL

Los Alamos, NM 87545

1 Kevin Watson

ANP Laboratory, MS W-82

The Boeing Company

Orgn. BXT60, P. O. Box 3707

Seattle, WA 98124

2 David Dolling

Dept. of Aerospace Engr. and

Engineering Mechanics

University of Texas at Austin

Austin, TX 78712-1085
NASA Langley Research Center

Attn: Jim Weilmuenster, MS 408A

Scott Berry, MS 408A

Peter Gnoffo, MS 408A

Doug Dwoyer, MS 105

Wally Sawyer, MS 119

Charles Miller, MS 408A

Hampton, VA 23681-0001

1 Linda Verdoom Powers

DP18

Dept. of Energy

19901 Germantown Road

Germantown, MD 20874

1 Chris Keane

DP 18

Dept. of Energy

19901 Germantown Road

Germantown, MD 20874

1 Rose Mary Baltrusaitis

Group XTA, MS B220

LANL

Los Alamos, NM 87545

1 Gary Carlson

ШNL

P. O. Box 808, L- 170

Livermore, CA 94551

1 John Erdos

GASL

77 Raynor Ave.

RonKon Koma, NY 11779

2 A. J. Smits

Dept. of Mechanical Engr.

Princeton University

Princeton, NJ 08544 


\begin{tabular}{|c|c|c|c|}
\hline 1 & MS 0429 & 2100 & R. D. Andreas \\
\hline 1 & MS 9005 & 2200 & J. B. Wright \\
\hline 1 & MS 0301 & 2400 & D. J. Rigali \\
\hline 1 & MS 0315 & 2411 & J.L. McDowell \\
\hline 1 & MS 0303 & 2414 & W. E. Williamson \\
\hline 1 & MS 0149 & 4000 & D. L. Hartley \\
\hline 1 & MS 0419 & 5412 & R. G. Easterling \\
\hline 1 & MS 1435 & 6200 & D. E. Arvizu \\
\hline 1 & MS 0708 & 6214 & H. M. Dodd \\
\hline 1 & MS 0736 & 6400 & N. R. Ortiz \\
\hline 1 & MS 0747 & 6412 & A. L. Camp \\
\hline 1 & MS 0742 & 6414 & J. E. Kelly \\
\hline 1 & MS 1345 & 6416 & P. A. Davis \\
\hline 1 & MS 0739 & 6421 & K. D. Bergeron \\
\hline 1 & MS 1139 & 6423 & K. D. Reil \\
\hline 1 & MS 1139 & 6423 & M. M. Pilch \\
\hline 1 & MS 9054 & 8300 & W. J. McLean \\
\hline 2 & MS 9042 & 8345 & C. M. Hartwig \\
\hline 1 & MS 9056 & 8351 & L. A. Rahn \\
\hline 1 & MS 9055 & 8353 & F.P. Tully \\
\hline 1 & MS 9052 & 8361 & D. R. Hardesty \\
\hline 1 & MS 9053 & 8362 & R. W. Carling \\
\hline 1 & MS 9405 & 8700 & T. M. Dyer \\
\hline 1 & MS 9161 & 8717 & W. G. Wolfer \\
\hline 1 & MS 9042 & 8742 & E. P. Chen \\
\hline 1 & MS 9405 & 8743 & P. E. Nielan \\
\hline 1 & MS 9044 & 8746 & W. A. Kawahara \\
\hline 1 & MS 9003 & 8900 & D. L. Crawford \\
\hline 1 & MS 9011 & 8950 & J. C. Meza \\
\hline 2 & MS 0151 & 9000 & G. Yonas \\
\hline 1 & MS 0841 & 9100 & P. J. Hommert \\
\hline 1 & MS 0833 & 9103 & J. H. Biffle \\
\hline 1 & MS 0437 & 9104 & R. K. Thomas \\
\hline 1 & MS 0826 & 9111 & W. Hermina \\
\hline 1 & MS 0826 & 9111 & D. K. Gartling \\
\hline 1 & MS 0826 & 9111 & S. N. Kempka \\
\hline 1 & MS 0826 & 9111 & M. A. Payne \\
\hline 1 & MS 0834 & 9112 & A. C. Ratzel \\
\hline 1 & MS 0835 & 9113 & T. C. Bickel \\
\hline 1 & MS 0835 & 9113 & B. F. Blackwell \\
\hline 1 & MS 0835 & 9113 & D. W. Kuntz \\
\hline 1 & MS 0835 & 9113 & V. J. Romero \\
\hline 1 & MS 0827 & 9114 & R. Griffith \\
\hline 1 & MS 0827 & 9114 & A. S. Geller \\
\hline 1 & MS 0825 & 9115 & W. H. Rutledge \\
\hline
\end{tabular}




\begin{tabular}{|c|c|c|}
\hline \multirow{2}{*}{$\begin{array}{l}\text { MS } 0825 \\
\text { MS } 0825\end{array}$} & 9115 & D.P. Aeschliman \\
\hline & 9115 & V. A. Amatucci \\
\hline MS 0825 & 9115 & R. S. Baty \\
\hline MS 0825 & 9115 & F. G. Blottner \\
\hline MS 0825 & 9115 & K. V. Chavez \\
\hline MS 0825 & 9115 & W. T. Gutierrez \\
\hline MS 0825 & 9115 & B. Hassan \\
\hline MS 0825 & 9115 & M. W. Kniskern \\
\hline MS 0825 & 9115 & A. R. Lopez \\
\hline MS 0825 & 9115 & W. L. Oberkampf \\
\hline MS 0825 & 9115 & J. L. Payne \\
\hline MS 0825 & 9115 & T. M. Sterk \\
\hline MS 0836 & 9116 & C. W. Peterson \\
\hline MS 0836 & 9116 & S.P. Burns \\
\hline MS 0836 & 9116 & G. F. Homicz \\
\hline MS 0836 & 9116 & D. D. McBride \\
\hline MS 0836 & 9116 & J. H. Strickland \\
\hline MS 0836 & 9116 & W. P. Wolfe \\
\hline MS 0443 & 9117 & H.S. Morgan \\
\hline MS 0321 & 9200 & W.J. Camp \\
\hline MS 0828 & 9209 & E. D. Gorham \\
\hline MS 1111 & 9225 & G. S. Heffelfinger \\
\hline MS 0819 & 9231 & J.S. Peery \\
\hline MS 0819 & 9231 & T. G. Trucano \\
\hline MS 0820 & 9232 & P. Yarrington \\
\hline MS 0439 & 9234 & D. R. Martinez \\
\hline MS 0439 & 9234 & K. F. Alvin \\
\hline MS 0439 & 9234 & M. S. Eldred \\
\hline MS 0439 & 9234 & J. R. Red-Horse \\
\hline MS 1165 & 9300 & J. E. Powell \\
\hline MS 1179 & 9341 & $\begin{array}{l}\text { J.R. Lee } \\
\text { TF Luera }\end{array}$ \\
\hline MS 1146 & $\begin{array}{l}9363 \\
9700\end{array}$ & $\begin{array}{l}\text { T. F. Luera } \\
\text { K. G. McCaughey }\end{array}$ \\
\hline MS 0865 & 9735 & J. L. Moya \\
\hline MS 0557 & 9741 & T.J. Baca \\
\hline MS 0428 & 12300 & W. C. Nickell \\
\hline MS 0829 & 12303 & K. V. Diegert \\
\hline MS 0829 & 12303 & B. M. Rutherford \\
\hline MS 9018 & $8940-2$ & Central Technical Files \\
\hline MS 0899 & 4414 & Technical Library \\
\hline MS 0619 & 12690 & $\begin{array}{l}\text { Review \& Approval Desk } \\
\text { For DOE/OSTI }\end{array}$ \\
\hline & & $-35-$ \\
\hline
\end{tabular}


